\title{
2013 E. H. Moore Prize
}

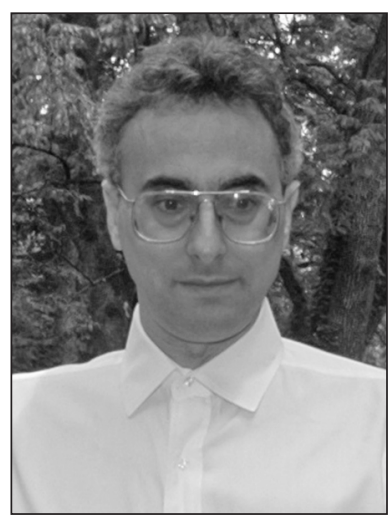

Michael J. Larsen

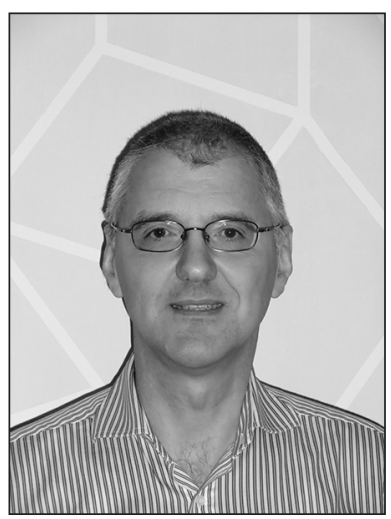

Richard Pink
Michael J. LARSEN and RICHARD PINK received the 2013 E. H. Moore Research Article Prize at the 119th Annual Meeting of the AMS in San Diego, California, in January 2013.

\section{Citation}

The 2013 E. H. Moore Research Prize is awarded to Michael J. Larsen and Richard Pink for their article "Finite subgroups of algebraic groups", Journal of the American Mathematical Society 24 (2011), no. $4,1105-1158$. The article provides a conceptual proof of an approximation to the classification of finite simple groups, using methods that are independent of the classification. Specifically, the article generalizes a fundamental result of Camille Jordan (1878) that concerns finite subgroups of $\mathrm{GL}_{n}$ over a field of characteristic 0 ; Jordan's theorem states that each such subgroup has a normal abelian subgroup of index bounded by a constant that depends only on $n$. Larsen and Pink work with arbitrary fields; they allow positive characteristic. Their situation is visibly more complicated than that studied by Jordan: consider, for example, what happens over a finite field, where the ambient group $\mathrm{GL}_{n}$ is finite and close to being simple. The authors prove, in particular, that finite simple subgroups of $\mathrm{GL}_{n}$ are either small (in terms of $n$ ) or are of Lie type.

Experts spoke of the impact of this article, praising the methods of the article as well as its conclusions. One report ended, "This was a tour de force, and I cannot think of a better choice for the E. H. Moore Prize."

\section{Biographical Sketches}

Michael Larsen graduated from Harvard College in 1984 and received a Ph.D. in mathematics from Princeton University in 1988. He has worked at the Institute for Advanced Study (1988-1990), the University of Pennsylvania (1990-1997), and the Uni-

DOI: http://dx.doi.org/10.1090/noti966 versity of Missouri (1997-1998) before coming to Indiana University, where he is now Distinguished Professor of Mathematics.

Richard Pink received his Ph.D. in mathematics from Bonn University in 1989 under the supervision of Professor Dr. G. Harder. An invited lecturer to the 2002 ICM in Beijing, China, he has taught at Bonn University, Harvard University, the MaxPlanck-Institut für Mathematik, and Mannheim University. Currently, he is teaching at ETH Zürich, where he served as chairman of the Department of Mathematics from 2004-2006. His research interests include number theory and arithmetic geometry, specifically the arithmetic of Shimura varieties, the topological-geometric nature of the Lefschetz trace formula, Drinfeld modules and their generalizations, and motives over function fields, including the arithmetic of the associated Galois representations. In 1996, after some preliminary sketches, he commissioned a master carpenter to design and build a fancy modular cabinet for his house (http://www.math.ethz. ch/ pink/ModularCabinet/cabinet.html); the design is based upon the action of the modular group on the Poincaré half-plane, an important idea that underlies much of his work.

\section{Joint Response from Michael J. Larsen and Richard Pink}

We would like to express our deep appreciation to the American Mathematical Society for this unexpected honor. At the time that we wrote the original draft of our paper (1998), the quasi-thin case of the classification of finite simple groups remained open. We wanted a weak version of the classification theorem that could be used for subgroups of $\mathrm{GL}_{n}$, for instance for Galois representations associated to Drinfeld modules or for strong approximation in the spirit of Boris Weisfeiler's work. We were inspired by the beautiful paper of Madhav Nori on subgroups of $\mathrm{GL}_{n}\left(\mathbb{F}_{p}\right)$ and were very glad to succeed using only the comparatively soft methods of algebraic geometry.

\section{About the Prize}

The Moore Prize is awarded every three years for an outstanding research article that appeared in one of the primary AMS research journals: Journal of the AMS, Proceedings of the AMS, Transactions of 
the AMS, AMS Memoirs, Mathematics of Computation, Electronic Journal of Conformal Geometry and Dynamics, or Electronic Journal of Representation Theory. The article must have appeared during the six calendar years ending a full year before the meeting at which the prize is awarded. The prize carries a cash award of US\$5,000.

The prize honors the extensive contributions of E. H. Moore (1862-1932) to the AMS. Moore founded the Chicago section of the AMS, served as the Society's sixth president (1901-1902), delivered the Colloquium Lectures in 1906, and founded and nurtured the Transactions of the AMS.
The Moore Prize is awarded by the AMS Council acting on the recommendation of a selection committee. For the 2013 prize, the members of the selection committee were: Sergiu Klainerman, Howard Masur, Bjorn Poonen, Kenneth A. Ribet, and Ulrike L. Tillman.

The previous recipients of the Moore Prize are Mark Haiman (2004), Ivan Shestakov and Ualbai Umirbaev (2007), and Sorin Popa (2010).

-Elaine Kehoe

\section{Robbins Prize}

AlEXANDER RAZBOROV received the David P. Robbins Prize at the 119th Annual Meeting of the AMS in San Diego, California, in January 2013.

\section{Citation}

The 2013 David P. Robbins Prize is awarded to Alexander Razborov of the University of Chicago for his paper "On the minimal density of triangles in graphs", Combinatorics, Probability and Computing 17 (2008), no. 4, 603-618, and for introducing a new powerful method, flag algebras, to solve problems in extremal combinatorics.

Razborov solves an old extremal problem about the minimum possible number of triangles in a graph with $n$ vertices and $m$ edges. The origin of this problem goes back more than one hundred years to one of the oldest results in extremal combinatorics, by Mantel, who proved that any such graph with more than $n^{2} / 4$ edges must have a triangle. This leads to the natural question of how many such triangles (as a function of the number of edges) should exist. Although the problem has been studied by leading combinatorialists for decades, it remained open until its recent solution by Razborov.

The paper by Razborov not only settled a longstanding open problem; much more importantly, it introduced a new method, called flag algebra calculus, for attacking a large class of extremal questions. This method was originally invented by Razborov to study the triangle density problem and was developed in full generality in his closely related paper, "Flag algebras", Journal of Symbolic Logic 72 (2007), no. 4, 1239-1282.

The solution of many extremal problems requires finding inequalities involving densities of small subgraphs of large graphs. Until recently this

DOI: http://dx.doi.org/10.1090/noti969 was done by ingenuity and the trialand-error method. Remarkably, the work of Razborov gives a systematic approach to these arguments. His flag algebra calculus provides a formalism through which the problem of finding relations between subgraph densities can be reduced to a semidefinite programming (SDP) problem. This in turn enables the use of computers to find solutions, with rigorous proofs, to problems in extremal combinatorics. This method already had a great impact on the area, and it has been used to settle a number of longstanding open problems in extremal

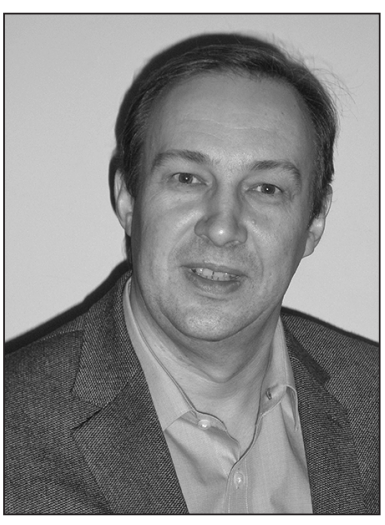

Alexander Razborov graph theory.

\section{Biographical Sketch}

Alexander Razborov was born in 1963 in the small Siberian town of Belovo. He received his B.Sc. in mathematics from Moscow State University and his Ph.D. from the Steklov Mathematical Institute (Moscow). Currently, he is an Andrew MacLeish Distinguished Service Professor at the Department of Computer Science at the University of Chicago, with part-time appointments at the Steklov Mathematical Institute and Toyota Technological Institute at Chicago. He received the Rolf Nevanlinna Prize in 1990 and the Gödel Prize in 2007, was an invited speaker at the ICM in Berkeley (1986), and was elected as a corresponding member of the Russian Academy of Sciences in 2000. His research spans several areas in theoretical computer science, including computational complexity, proof complexity, quantum computing, and computational complexity, as well as related mathematical areas, notably discrete mathematics and combinatorial group theory. 